\title{
ON THE COHOMOLOGY OF TORI OVER LOCAL FIELDS WITH PERFECT RESIDUE FIELD
}

\author{
ALESSANDRA BERTAPELLE AND CRISTIAN D. GONZÁLEZ-AVILÉS
}

\begin{abstract}
If $T$ is an algebraic torus defined over a discretely valued field $K$ with perfect residue field $k$, we relate the $K$-cohomology of $T$ to the $k$-cohomology of certain objects associated to $T$. When $k$ has cohomological dimension $\leq 1$, our results have a particularly simple form and yield, more generally, isomorphisms between Borovoi's abelian $K$-cohomology of a reductive group $G$ over $K$ and the $k$-cohomology of a certain quotient of the algebraic fundamental group of $G$.
\end{abstract}

\section{INTRODUCTION}

Let $A$ be a complete discrete valuation ring with field of fractions $K$ and perfect residue field $k$. Let $\bar{k}$ and $K^{\text {sep }}$ be fixed separable algebraic closures of $k$ and $K$, respectively, and let $g$ and $\mathcal{G}$ denote the corresponding absolute Galois groups. Further, let $K^{\mathrm{nr}}$ denote the maximal unramified extension of $K$ in $K^{\text {sep }}$ and let $I=\operatorname{Gal}\left(K^{\mathrm{sep}} / K^{\mathrm{nr}}\right)$ be the inertia subgroup of $\mathcal{G}$. Then there exists a canonical isomorphism of groups $\mathcal{G} / I=g$. If $M$ is a $\mathcal{G}$-module, $M_{I}$ will denote the g-module of $I$-coinvariants of $M$. Now let $T$ be a $K$-torus and let $X^{*}(T)$ (respectively, $X_{*}(T)$ ) denote the $\mathcal{G}$-module of characters (respectively, cocharacters) of $T$. Let $\mathscr{T}$ denote the Néron model of $T$ over $S:=\operatorname{Spec} A$ and let $i$ : Spec $k \rightarrow S$ be the canonical closed immersion. The group of components of $\mathscr{T}$, i.e., the (continuous) g-module $\phi(T)$ which corresponds to the étale $k$-sheaf $i^{*}\left(\mathscr{T} / \mathscr{T}^{0}\right)$, was described by Xarles in [31] in terms of $X^{*}(T)$. The description given in [31] is simple when $\phi(T)$ is either torsion or torsion-free, but this is not the case in general. When $k$ is finite, a much simpler description of $\phi(T)$ was obtained by Bitan in [2, (3.1)], who showed the existence of an isomorphism of $g$-modules $\phi(T) \simeq X_{*}(T)_{I}$ for such $k$. We generalize Bitan's beautiful formula to the case of any perfect field $k$. That is, we prove

Theorem 1.1. There exists a canonical isomorphism of g-modules

$$
\phi(T) \stackrel{\sim}{\rightarrow} X_{*}(T)_{I} .
$$

Date: October 8, 2018.

2010 Mathematics Subject Classification. Primary 20G25; Secondary 11S25, 12 G05.

Key words and phrases. Local fields with perfect residue field, Algebraic torus, cohomology, Néron model, group of components.

C. G-A. was partially supported by Fondecyt grant 1120003. 
We note that Bitan [2, (3.1)] obtained his formula by combining work of Kottwitz [19, §7.2] and of Haines and Rapoport [24, Appendix]. Since [24, Appendix] depends on Bruhat-Tits, so does the proof of [2, (3.1)]. Although Bitan's method can be extended to yield a proof of his formula for any perfect field $k$, in this paper we have chosen to generalize his formula by means of an explicit and functorial construction of the isomorphism of Theorem 1.1 independently of Bruhat-Tits theory.

The above theorem has a number of (immediate) consequences which shed new light on the present subject. For example, the theorem implies that the functor $\phi(-)$ transforms short exact sequences of $K$-tori into 6 -term exact sequences of $g$-modules. See Proposition 3.8 for the precise statement.

In Section 4 we use Theorem 1.1 to relate the $K$-cohomology of $T$ to the $k$ cohomology of $X_{*}(T)_{I}$. When $k$ has cohomological dimension $\leq 1$, our results have the following simple form.

Theorem 1.2 (=Theorem 4.5). Assume that $k$ has cohomological dimension $\leq 1$. Then, for $r=1$ and 2, there exist canonical isomorphisms of abelian groups

$$
H^{r}(K, T) \simeq H^{r}\left(k, X_{*}(T)_{I}\right)
$$

If $r \geq 3$, the groups $H^{r}(K, T)$ vanish.

In Section 5 we generalize the above theorem from $K$-tori to arbitrary connected reductive algebraic $K$-groups $G$. More precisely, let $\pi_{1}(G)$ be the algebraic fundamental group of $G$. Then the following holds.

Theorem 1.3 (=Theorem 5.1). Assume that $k$ has cohomological dimension $\leq 1$ and let $G$ be a connected reductive algebraic group over $K$. Then, for $r=1$ and 2, there exist isomorphisms of abelian groups

$$
H_{\mathrm{ab}}^{r}\left(K_{\mathrm{fl}}, G\right) \simeq H^{r}\left(k, \pi_{1}(G)_{I}\right) .
$$

If $r \geq 3$, the groups $H_{\mathrm{ab}}^{r}\left(K_{\mathrm{fl}}, G\right)$ vanish.

Corollary 1.4 (=Corollary 5.2). Assume that $k$ has cohomological dimension $\leq 1$ and let $G$ be a connected reductive algebraic group over $K$. Then there exists a bijection of pointed sets

$$
H^{1}(K, G) \simeq H^{1}\left(k, \pi_{1}(G)_{I}\right)
$$

In particular, $H^{1}(K, G)$ can be endowed with an abelian group structure.

\section{ACKNOWLEDGEMENTS}

We are very grateful to Mikhail Borovoi for some valuable suggestions and for sending us the proof of Lemma 5.4. We also thank Rony Bitan for helpful comments. 


\section{Preliminaries}

2.1. The Basic Setting. We keep the notation introduced in the previous section. If $K$ is any field and $T$ is a $K$-torus, let $\underline{X}^{*}(T):=\underline{\operatorname{Hom}}_{K}\left(T, \mathbb{G}_{m, K}\right)$ be the étale $K$ sheaf of characters of $T$ and set $X^{*}(T)=\underline{X}^{*}(T)\left(K^{\mathrm{sep}}\right)$. Note that, since $\underline{X}^{*}(T)$ is locally constant, it is represented by a unique (commutative) étale $K$-group scheme. See [29, Proposition II.9.2.3, p. 153]. There exists a canonical isomorphism of étale $K$-sheaves

$$
T=\underline{\operatorname{Hom}}_{K}\left(\underline{X}^{*}(T), \mathbb{G}_{m, K}\right) .
$$

See, for example, [5, Theorem 0.3.12, p. 11]. In particular, if $L / K$ is any Galois subextension of $K^{\mathrm{sep}} / K$ which splits $T$, then there exists a canonical isomorphism of $\operatorname{Gal}(L / K)$-modules

$$
T(L)=\operatorname{Hom}\left(X^{*}(T), L^{*}\right)
$$

Now let $\underline{X}_{*}(T):=\underline{\operatorname{Hom}}_{K}\left(\mathbb{G}_{m, K}, T\right)$ be the étale $K$-sheaf of cocharacters of $T$ and set $X_{*}(T)=\underline{X}_{*}(T)\left(K^{\mathrm{sep}}\right)$. We will identify $\underline{X}_{*}(T)$ with $\underline{X}^{*}(T)^{\vee}:=\underline{\operatorname{Hom}}_{K}\left(\underline{X}^{*}(T), \mathbb{Z}_{K}\right)$ and $X_{*}(T)$ with $X^{*}(T)^{\vee}=\operatorname{Hom}\left(X^{*}(T), \mathbb{Z}\right)$.

Let $S$ be a scheme and let $(\operatorname{Sch} / S)_{\text {ét }}^{\sim}$ be the category of sheaves of sets on the étale site over $S$. By [10, I, 1.1] (see also [29, Theorem II.3.1.2, p. 97]), the functor $h_{S}:(\operatorname{Sch} / S) \rightarrow(\operatorname{Sch} / S)_{\text {ét }}^{\sim}, Y \mapsto \operatorname{Hom}_{S}(-, Y)$, is fully faithful. If $Y$ is an $S$-scheme, we will identify $Y$ with $h_{S}(Y)$, i.e., with the étale sheaf it represents. If $S=\operatorname{Spec} F$, where $F$ is a field, then

$$
h_{F}:(\mathrm{Sch} / F) \rightarrow(\mathrm{Sch} / F)_{\text {ét }}^{\sim}, \quad Y \mapsto \operatorname{Hom}_{F}(-, Y),
$$

is, in fact, an equivalence of categories by [21, p. 54, last paragraph].

Now recall $S=\operatorname{Spec} A$ and $i:$ Spec $k \rightarrow S$. Let $j:$ Spec $K \rightarrow S$ be the canonical morphism. The Néron model $\mathscr{T}$ of $T$ over $S$ is a smooth and separated $S$-group scheme which represents the sheaf $j_{*} T$ on the étale (in fact, small smooth) site over S. See [4, Proposition 10.1.6, p. 292]. With one exception (namely, in Proposition (2.2) (i)), we will regard $j_{*} T$ as an étale sheaf on $S$ and identify it with (the étale sheaf on $S$ represented by) $\mathscr{T}$. Thus we may write $j_{*} T=\mathscr{T}$. The identity component $\mathscr{T}^{0}$ of $\mathscr{T}$ is a smooth affine $S$-group scheme of finite type. See [20, Proposition 3, p. 18] and [10, $\mathrm{VI}_{\mathrm{B}}$, Corollary 3.6]. Now set $\mathscr{T}_{s}=\mathscr{T} \times_{S}$ Spec $k$ and $\mathscr{T}_{s}^{0}=\mathscr{T}^{0} \times_{S}$ Spec $k$. Then $\mathscr{T}_{s}^{0}$ is a smooth, connected and affine $k$-group scheme of finite type (see [18, Proposition 17.3.3(iii)], [17, Proposition 1.6.2(iii)] and [16, Proposition 6.3.4(iii), p. 304]). By [11, II, §5, no.1, Proposition 1.8, p. 237], the étale $k$-group scheme $\pi_{0}\left(\mathscr{T}_{s}\right):=\mathscr{T}_{s} / \mathscr{T}_{s}^{0}$ has the following universal property: if $E$ is an étale $k$-group scheme and $\mathscr{T}_{s} \rightarrow E$ is a homomorphism of $k$-group schemes, then there exists a unique homomorphism of $k$-group schemes $\pi_{0}\left(\mathscr{T}_{s}\right) \rightarrow E$ such that the following 
diagram commmutes

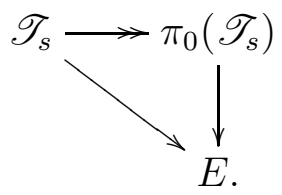

The $k$-group schemes $\mathscr{T}_{s}$ and $\mathscr{T}_{s}^{0}$ represent the étale $k$-sheaves $i^{*} \mathscr{T}$ and $i^{*} \mathscr{T}^{0}$, i.e., we may write $i^{*} \mathscr{T}=\mathscr{T}_{s}$ and $i^{*} \mathscr{T}^{0}=\mathscr{T}_{s}^{0}$ as étale sheaves on Spec $k$. Then the étale $k$-sheaf $\phi(T):=i^{*}\left(\mathscr{T} / \mathscr{T}^{0}\right)$ is represented by $\pi_{0}\left(\mathscr{T}_{s}\right)$, i.e,

$$
\phi(T)=\pi_{0}\left(\mathscr{T}_{s}\right)
$$

as étale sheaves on Spec $k$. We will often identify the étale sheaf $\phi(T)$ with the $g$ module $\phi(T)(\bar{k})=\pi_{0}\left(\mathscr{T}_{s}\right)(\bar{k})$ (see [29, Corollary II.2.2(i), p. 94]). By [5, Theorem 2.3 .2 , p. 51], $\phi(T)$ is a finitely generated $g$-module. Now, since $j^{*}\left(\mathscr{T} / \mathscr{T}^{0}\right)=0$, there exists a canonical isomorphism of étale sheaves $i_{*} \phi(T)=\mathscr{T} / \mathscr{T}^{0}$ (see [29, proof of Theorem II.8.1.2, p. 135]). Thus there exists a canonical exact sequence of étale sheaves on $S$

$$
0 \rightarrow \mathscr{T}^{0} \rightarrow \mathscr{T} \rightarrow i_{*} \phi(T) \rightarrow 0
$$

If $T=\mathbb{G}_{m, K}$, the preceding sequence is

$$
0 \rightarrow \mathbb{G}_{m, S} \rightarrow j_{*} \mathbb{G}_{m, K} \rightarrow i_{*} \mathbb{Z}_{k} \rightarrow 0,
$$

where the right-hand nontrivial morphism is induced by the valuation $v: K^{*} \rightarrow \mathbb{Z}$. See [4, §10.1, Example 5, p. 291].

2.2. Group Cohomology. Let $J$ be a finite group. We will write $|J|$ for its order and $\mathfrak{A}_{J}$ for the augmentation ideal of $\mathbb{Z}[J]$, i.e., the kernel of the homomorphism $\mathbb{Z}[J] \rightarrow \mathbb{Z}, \Sigma n_{\sigma} \sigma \mapsto \Sigma n_{\sigma}$. If $M$ is a finitely generated (left) $J$-module, $M_{J}:=M / \mathfrak{A}_{J} M$ is the largest quotient of $M$ on which $J$ acts trivially. Let $M^{\vee}=$ $\operatorname{Hom}_{\mathbb{Z}}(M, \mathbb{Z})$ be the linear dual of $M$. Then $M^{\vee}$ has a natural structure of $J$-module by [7, (1), p. 238] and $M^{\vee}=\left(M / M_{\text {tors }}\right)^{\vee}$ is either zero or $\mathbb{Z}$-free. We have (see [7, pp. 238-240])

$$
\left(M^{\vee}\right)^{J}=\left(M_{J}\right)^{\vee}
$$

and

$$
M^{\vee \vee}=M / M_{\text {tors }}
$$

In particular, $M^{\vee \vee}=M$ if $M$ is $\mathbb{Z}$-free. The kernel of the canonical norm map

$$
N: M \rightarrow M^{J}, \quad m \mapsto \sum_{\sigma \in J} \sigma m
$$


will be denoted by ${ }_{N} M$. Now recall that the Tate cohomology groups $\widehat{H}^{r}(J, M)$, for $r \in \mathbb{Z}$, are defined as follows: $\widehat{H}^{r}(J, M)=H^{r}(J, M)$ if $r \geq 1, \widehat{H}^{r}(J, M)=$ $H_{-r-1}(J, M)$ if $r \leq-2$ and

$$
\begin{aligned}
\widehat{H}^{0}(J, M) & =M^{J} / N M, \\
\widehat{H}^{-1}(J, M) & ={ }_{N} M / \mathfrak{A}_{J} M .
\end{aligned}
$$

Note that, if $J$ acts trivially on $M$ and $M$ is $\mathbb{Z}$-free, then ${ }_{N} M=0$ and therefore $\widehat{H}^{-1}(J, M)=0$. Further, by [7, Chapter XII, Proposition 2.5, p. 236, and Exercise 3, p. 263], $\widehat{H}^{r}(J, M)$ is a finite group which is annihilated by $|J|$ for every $r \in \mathbb{Z}$.

Next, if $M$ is a finitely generated abelian group, we will write $M^{D}=\operatorname{Hom}_{\mathbb{Z}}(M, \mathbb{Q} / \mathbb{Z})$. If $M$ is free, then $M^{D}=M^{\vee} \otimes_{\mathbb{Z}} \mathbb{Q} / \mathbb{Z}$ by [7, Chapter XII, beginning of $\S 3$, pp. 237238 .

Now assume that $M$ is a $\mathbb{Z}$-free and finitely generated $J$-module. Since $M^{D}=$ $M^{\vee} \otimes_{\mathbb{Z}} \mathbb{Q} / \mathbb{Z}$ as noted above, the short exact sequence $0 \rightarrow \mathbb{Z} \rightarrow \mathbb{Q} \rightarrow \mathbb{Q} / \mathbb{Z} \rightarrow 0$ induces a short exact sequence of $J$-modules $0 \rightarrow M^{\vee} \rightarrow M^{\vee} \otimes_{\mathbb{Z}} \mathbb{Q} \rightarrow M^{D} \rightarrow 0$. The latter sequence induces, in turn, a canonical isomorphism of abelian groups $\widehat{H}^{r-1}\left(J, M^{D}\right)=\widehat{H}^{r}\left(J, M^{\vee}\right)$ for every $r \in \mathbb{Z}$. On the other hand, by [7, Chapter XII, $\S 6$, Theorem 6.4, p. 249], $\widehat{H}^{r-1}\left(J, M^{D}\right)$ is canonically isomorphic to $\widehat{H}^{-r}(J, M)^{D}$. Thus, for every $r \in \mathbb{Z}$, there exists a canonical isomorphism of finite abelian groups

$$
\widehat{H}^{r}(J, M)^{D}=\widehat{H}^{-r}\left(J, M^{\vee}\right) .
$$

Lemma 2.1. Let $M$ be a $\mathbb{Z}$-free and finitely generated $J$-module.

(i) There exists a canonical exact sequence of abelian groups

$$
0 \rightarrow H^{1}(J, M)^{D} \rightarrow\left(M^{\vee}\right)_{J} \rightarrow\left(M^{J}\right)^{\vee} \rightarrow 0 .
$$

(ii) If $M^{J} \neq M$, then $M / M^{J}$ is $\mathbb{Z}$-free and $\left(M / M^{J}\right)^{J}=0$.

Proof. Applying the snake lemma to the exact commutative diagram

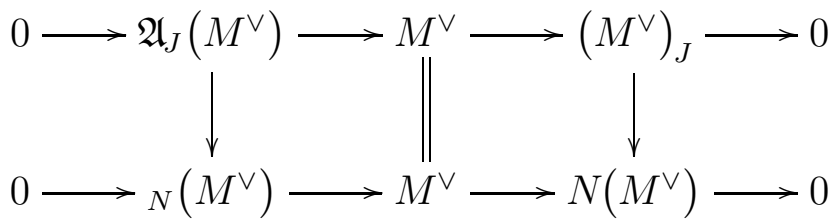

and using (2.10), we obtain an exact sequence

$$
0 \rightarrow H^{1}(J, M)^{D} \rightarrow\left(M^{\vee}\right)_{J} \rightarrow N\left(M^{\vee}\right) \rightarrow 0 .
$$

Now, since $N\left(M^{\vee}\right)$ is a subgroup of the $\mathbb{Z}$-free group $M^{\vee}$ and $H^{1}(J, M)^{D}$ is torsion, the latter sequence induces, by (2.7) and (2.8), canonical isomorphisms

$$
N\left(M^{\vee}\right)=\left(M^{\vee}\right)_{J} /\left(M^{\vee}\right)_{J, \text { tors }}=\left(\left(M^{\vee}\right)_{J}\right)^{\vee \vee}=\left(\left(M^{\vee \vee}\right)^{J}\right)^{\vee}=\left(M^{J}\right)^{\vee} \text {. }
$$

Assertion (i) is now clear. 
To prove (ii), we first note that, since $M^{J}$ is $\mathbb{Z}$-free, (2.12) yields a canonical isomorphism $N\left(M^{\vee}\right)^{\vee}=\left(M^{J}\right)^{\vee \vee}=M^{J}$. Therefore, taking the linear dual of the bottom row of the preceding diagram, we obtain an isomorphism

$$
\left({ }_{N}\left(M^{\vee}\right)\right)^{\vee}=M / M^{J} \text {. }
$$

Consequently, if $M^{J} \neq M$, then $M / M^{J}$ is $\mathbb{Z}$-free. Further, it follows from (2.11) that, if $M^{\prime}$ is a $\mathbb{Z}$-free and finitely generated $J$-module such that $N M^{\prime}=0$, then $M_{J}^{\prime}$ is torsion. Thus $\left({ }_{N}\left(M^{\vee}\right)\right)_{J}$ is torsion. Now, by (2.7) and (2.13)

$$
\left(M / M^{J}\right)^{J}=\left(\left({ }_{N}\left(M^{\vee}\right)\right)^{\vee}\right)^{J}=\left(\left({ }_{N}\left(M^{\vee}\right)\right)_{J}\right)^{\vee}=0,
$$

since $\left({ }_{N}\left(M^{\vee}\right)\right)_{J}$ is torsion.

2.3. A Canonical Resolution of $T$. Let $L$ be the minimal splitting field of $T$, i.e., the fixed field of the kernel of the canonical homomorphism $\mathcal{G} \rightarrow \operatorname{Aut}\left(X^{*}(T)\right)$. We will write $T_{L}$ for the (split) $L$-torus $T \times_{\text {Spec } K} \operatorname{Spec} L$. Let $J$ be the inertia subgroup of $\operatorname{Gal}(L / K)$. Then $I$ acts on the free and finitely generated $\mathbb{Z}$-module $X^{*}(T)$ through the finite quotient $J$ and (2.9) is a map

$$
N: X^{*}(T) \rightarrow X^{*}(T)^{J}
$$

Note that, since $H^{1}\left(I^{\prime}, X^{*}(T)\right)=\operatorname{Hom}\left(I^{\prime}, X^{*}(T)\right)=0$ for any subgroup $I^{\prime}$ of $I$ which acts trivially on $X^{*}(T)$ (as $I^{\prime}$ is torsion and $X^{*}(T)$ is torsion-free), the inflation-restriction exact sequence (see [25, VII, §6, Proposition 4, p. 117]) shows that $H^{1}\left(J, X^{*}(T)\right)=H^{1}\left(I, X^{*}(T)\right)$.

A $K$-torus $T$ is said to have multiplicative reduction if the special fiber $\mathscr{T}_{s}^{0}$ of $\mathscr{T}^{0}$ is a $k$-torus. An equivalent condition is that $I$ act trivially on $X^{*}(T)$, i.e., $T$ splits over $K^{\mathrm{nr}}$. If this is the case, then the $g$-module of characters of $\mathscr{T}_{s}^{0}$ is $X^{*}(T)$. See [23, Proposition-Definition 1.1, p. 462]. Further, there exists a canonical isomorphism of g-modules

$$
\phi(T) \stackrel{\sim}{\rightarrow} X_{*}(T) .
$$

See [5, Theorem 1.1.2, p. 29] and recall the identification $X^{*}(T)^{\vee}=X_{*}(T)$.

Proposition 2.2. Let $0 \rightarrow T_{1} \rightarrow T_{2} \rightarrow T_{3} \rightarrow 0$ be an exact sequence of $K$-tori. Assume that the following conditions hold:

(i) $\mathrm{R}^{1} j_{*} T_{1}=0$ for the smooth topology on $S$, and

(ii) $\phi\left(T_{1}\right)$ is torsion-free.

Then the induced sequence of $g$-modules $0 \rightarrow \phi\left(T_{1}\right) \rightarrow \phi\left(T_{2}\right) \rightarrow \phi\left(T_{3}\right) \rightarrow 0$ is exact.

Proof. By (i), the sequence of Néron models $0 \rightarrow \mathscr{T}_{1} \rightarrow \mathscr{T}_{2} \rightarrow \mathscr{T}_{3} \rightarrow 0$ is exact in the smooth topology. Thus, by [5, Theorem 2.3.1, p. 50], the induced sequence of g-modules $\phi\left(T_{1}\right) \rightarrow \phi\left(T_{2}\right) \rightarrow \phi\left(T_{3}\right) \rightarrow 0$ is exact. On the other hand, by [5, Theorem 2.3.4, p. 52], $\operatorname{Ker}\left[\phi\left(T_{1}\right) \rightarrow \phi\left(T_{2}\right)\right]$ is a finite $g$-submodule of $\phi\left(T_{1}\right)$, which is therefore zero by (ii). This completes the proof. 
Corollary 2.3. Let $0 \rightarrow T_{1} \rightarrow T_{2} \rightarrow T_{3} \rightarrow 0$ be an exact sequence of $K$-tori, where $T_{1}$ has multiplicative reduction. Then the induced sequence of g-modules $0 \rightarrow$ $\phi\left(T_{1}\right) \rightarrow \phi\left(T_{2}\right) \rightarrow \phi\left(T_{3}\right) \rightarrow 0$ is exact.

Proof. Since $T_{1}$ splits over $K^{\mathrm{nr}}, \mathrm{R}^{1} j_{*} T_{1}=0$ for the smooth topology on $S$ by [5, Corollary 4.2.6, p. 82]. On the other hand, by (2.15), $\phi\left(T_{1}\right) \simeq X_{*}\left(T_{1}\right)$, which is torsion-free. The corollary is now immediate from the proposition.

A $K$-torus $T$ is said to have unipotent reduction if the special fiber $\mathscr{T}_{s}^{0}$ of $\mathscr{T}^{0}$ is a unipotent $k$-group scheme.

Lemma 2.4. A $K$-torus $T$ has unipotent reduction if, and only if, $X^{*}(T)^{I}=0$.

Proof. By [23, proof of Theorem 1.3], $T$ has unipotent reduction if, and only if, $T$ contains no nontrivial $K$-subtorus having multiplicative reduction, i.e., $X^{*}(T)$ admits no free quotient on which $I$ (or, equivalently, $J$ ) acts trivially. Assume that the latter holds and recall the norm map (2.14). Since $N X^{*}(T)$ is a quotient of $X^{*}(T)$ with trivial $J$-action, we have $N X^{*}(T)=0$ and therefore $X^{*}(T)^{J}=$ $X^{*}(T)^{J} / N X^{*}(T)=\widehat{H}^{0}\left(J, X^{*}(T)\right)$ is a finite subgroup of the free group $X^{*}(T)$, i.e., $X^{*}(T)^{I}=X^{*}(T)^{J}=0$. Conversely, assume that $X^{*}(T)^{I}=0$ and let $Y$ be an $I$ submodule of $X^{*}(T)$ such that $I$ acts trivially on $X^{*}(T) / Y$. Then the $I$-cohomology sequence associated to $0 \rightarrow Y \rightarrow X^{*}(T) \rightarrow X^{*}(T) / Y \rightarrow 0$ shows that $X^{*}(T) / Y$ is isomorphic to a subgroup of the finite group $H^{1}(I, Y)$. In particular, it is not free.

What follows is an elaboration of [31, Lemma 2.13].

Let $T$ be any $K$-torus. The maximal quotient torus $T^{(m)}$ of $T$ having multiplicative reduction is the $K$-torus with character module $X^{*}(T)^{I}$, and the maximal subtorus $T_{(u)}$ of $T$ having unipotent reduction is the $K$-torus with character module $X^{*}(T) / X^{*}(T)^{I}$. This follows from Lemmas 2.1(ii) and 2.4 together with the fact that, if $Y$ is an $I$-submodule of $X^{*}(T)$ such that $X^{*}(T) / Y$ is free and $\left(X^{*}(T) / Y\right)^{I}=0$, then $X^{*}(T)^{I} \subset Y$. Now the exact sequence of $\mathcal{G}$-modules $0 \rightarrow X^{*}(T)^{I} \rightarrow X^{*}(T) \rightarrow X^{*}(T) / X^{*}(T)^{I} \rightarrow 0$ induces an exact sequence of $K$-tori

$$
0 \rightarrow T_{(u)} \rightarrow T \rightarrow T^{(m)} \rightarrow 0 .
$$

Recall now the minimal splitting field $L$ of $T$. The norm map $N_{L / K}: L^{*} \rightarrow$ $K^{*}$ induces an epimorphism of $K$-tori $R_{L / K}\left(T_{L}\right) \rightarrow T$ whose kernel is denoted by $R_{L / K}^{(1)}\left(T_{L}\right)$ and called the norm one torus associated to $T$. See [5, Theorem 0.4.4, p. 16]. Thus there exist canonical exact sequences

$$
0 \rightarrow R_{L / K}^{(1)}\left(T_{L}\right) \rightarrow R_{L / K}\left(T_{L}\right) \rightarrow T \rightarrow 0
$$

and

$$
0 \rightarrow R_{L / K}^{(1)}\left(T_{L}\right)_{(u)} \rightarrow R_{L / K}^{(1)}\left(T_{L}\right) \rightarrow R_{L / K}^{(1)}\left(T_{L}\right)^{(m)} \rightarrow 0
$$


where the latter sequence is the sequence (2.16) associated to the $K$-torus $R_{L / K}^{(1)}\left(T_{L}\right)$. Set

$$
P=R_{L / K}^{(1)}\left(T_{L}\right)^{(m)}
$$

and let $Q$ be the pushout of the canonical morphisms $R_{L / K}^{(1)}\left(T_{L}\right) \hookrightarrow R_{L / K}\left(T_{L}\right)$ and $R_{L / K}^{(1)}\left(T_{L}\right) \rightarrow R_{L / K}^{(1)}\left(T_{L}\right)^{(m)}=P$ appearing in (2.17) and (2.18), respectively. Thus there exists a canonical exact commutative diagram

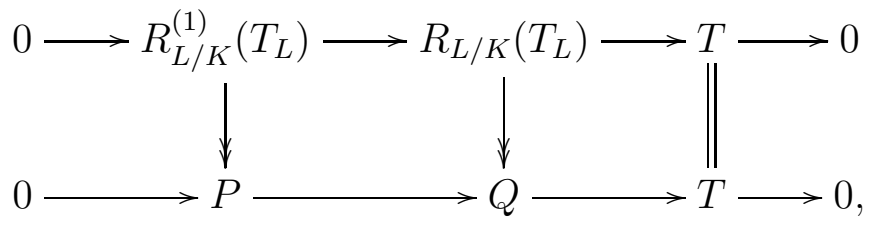

where the top row is (2.17). By (2.18), the kernel of the left-hand vertical map in the above diagram equals $R_{L / K}^{(1)}\left(T_{L}\right)_{(u)}$, which immediately yields the formula

$$
Q=R_{L / K}\left(T_{L}\right) / R_{L / K}^{(1)}\left(T_{L}\right)_{(u)}
$$

Thus there exists a canonical exact sequence of $K$-tori

$$
0 \rightarrow P \rightarrow Q \rightarrow T \rightarrow 0,
$$

where $P$ and $Q$ are given by (2.19) and (2.20), respectively. Since $P$ has multiplicative reduction, the following lemma is immediate from Corollary 2.3 .

Lemma 2.5. The canonical resolution (2.21) induces an exact sequence of g-modules

$$
0 \rightarrow \phi(P) \rightarrow \phi(Q) \rightarrow \phi(T) \rightarrow 0 .
$$

Lemma 2.6. The canonical resolution (2.21) induces an exact sequence of g-modules

$$
0 \rightarrow X_{*}(P) \rightarrow X_{*}(Q)_{I} \rightarrow X_{*}(T)_{I} \rightarrow 0 .
$$

Proof. The $J$-homology sequence associated to the short exact sequence of $J$-modules $0 \rightarrow X_{*}(P) \rightarrow X_{*}(Q) \rightarrow X_{*}(T) \rightarrow 0$ corresponding to (2.21) is

$$
\cdots \rightarrow H_{1}\left(J, X_{*}(Q)\right) \rightarrow H_{1}\left(J, X_{*}(T)\right) \rightarrow X_{*}(P)_{I} \rightarrow X_{*}(Q)_{I} \rightarrow X_{*}(T)_{I} \rightarrow 0 .
$$

Since $H_{1}\left(J, X_{*}(T)\right)$ is torsion and $X_{*}(P)_{I}=X_{*}(P)$ is torsion-free, the lemma follows.

Lemma 2.7. $H^{1}\left(I, X^{*}(Q)\right)=0$.

Proof. By [5, Theorem 0.4.3, p. 14, and proof of Theorem 0.4.4, p. 16], there exists a canonical isomorphism of $\mathcal{G}$-modules $X^{*}\left(R_{L / K}\left(T_{L}\right)\right)=\mathbb{Z}^{d}[\operatorname{Gal}(L / K)]$, where $d$ is the dimension of $T$. Thus (2.20) yields a canonical exact sequence of $\mathcal{G}$-modules

$$
0 \rightarrow X^{*}(Q) \rightarrow \mathbb{Z}^{d}[\operatorname{Gal}(L / K)] \rightarrow X^{*}\left(R_{L / K}^{(1)}\left(T_{L}\right)_{(u)}\right) \rightarrow 0
$$


Now, since $X^{*}\left(R_{L / K}^{(1)}\left(T_{L}\right)_{(u)}\right)^{J}=0$ by Lemma 2.4, the $J$-cohomology sequence associated to the above short exact sequence yields an injection

$$
H^{1}\left(I, X^{*}(Q)\right)=H^{1}\left(J, X^{*}(Q)\right) \hookrightarrow H^{1}\left(J, \mathbb{Z}^{d}[\operatorname{Gal}(L / K)]\right) .
$$

Finally, since $\mathbb{Z}^{d}[\mathrm{Gal}(L / K)]$ is a free (right) $\mathbb{Z}^{d}[J]$-module of finite rank, the latter cohomology group vanishes by Shapiro's lemma (see [30, Lemma 6.3.2, p. 171]), and this completes the proof.

\section{Proof of Theorem 1.1}

By Lemma 2.1(i), there exists a canonical exact sequence of $g$-modules

$$
0 \longrightarrow H^{1}\left(I, X^{*}(T)\right)^{D} \longrightarrow X_{*}(T)_{I} \stackrel{q_{T}}{\longrightarrow}\left(X^{*}(T)^{I}\right)^{\vee} \longrightarrow 0 .
$$

We will write $\underline{X}_{*}(T)_{I}$ and $\underline{X}^{*}(T)^{I}$, respectively, for the étale $k$-sheaves that correspond to the continuous g-modules $X_{*}(T)_{I}$ and $X^{*}(T)^{I}$ (see [29, Corollary II.2.2(i), p. 94]).

Lemma 3.1. There exists a canonical exact sequence of étale sheaves on $S$

$$
0 \rightarrow \underline{\operatorname{Hom}}_{S}\left(j_{*} \underline{X}^{*}(T), \mathbb{G}_{m, S}\right) \rightarrow \mathscr{T} \rightarrow i_{*}\left(\underline{X}^{*}(T)^{I}\right)^{\vee} \rightarrow 0 .
$$

Proof. Since $\underline{\operatorname{Ext}}_{S}^{1}{ }_{\text {ét }}\left(j_{*} \underline{X}^{*}(T), \mathbb{G}_{m, S}\right)=0$ by [5, Theorem B.3, p. 131], (2.6) induces an exact sequence of étale sheaves on $S$

$0 \rightarrow \underline{\operatorname{Hom}}_{S}\left(j_{*} \underline{X}^{*}(T), \mathbb{G}_{m, S}\right) \rightarrow \underline{\operatorname{Hom}}_{S}\left(j_{*} \underline{X}^{*}(T), j_{*} \mathbb{G}_{m, K}\right) \rightarrow \underline{\operatorname{Hom}}_{S}\left(j_{*} \underline{X}^{*}(T), i_{*} \mathbb{Z}_{k}\right) \rightarrow 0$.

Now, since $\underline{X}^{*}(T)=j^{*} j_{*} \underline{X}^{*}(T)$ by [29, Proposition II.8.1.1, p. 134] and $i^{*} j_{*} \underline{X}^{*}(T)=$ $\underline{X}^{*}(T)^{I}$ by [21, Example II.3.12, p. 75], (2.1) and [21, Exercise II.3.22(a), p. 80] yield canonical isomorphisms of étale sheaves on $S$

$$
\underline{\operatorname{Hom}}_{S}\left(j_{*} \underline{X}^{*}(T), j_{*} \mathbb{G}_{m, K}\right)=j_{*} \underline{\operatorname{Hom}}_{K}\left(j^{*} j_{*} \underline{X}^{*}(T), \mathbb{G}_{m, K}\right)=j_{*} T=\mathscr{T}
$$

and

$$
\underline{\operatorname{Hom}}_{S}\left(j_{*} \underline{X}^{*}(T), i_{*} \mathbb{Z}_{k}\right)=i_{*} \underline{\operatorname{Hom}}_{k}\left(i^{*} j_{*} \underline{X}^{*}(T), \mathbb{Z}_{k}\right)=i_{*}\left(\underline{X}^{*}(T)^{I}\right)^{\vee}
$$

The lemma is now clear.

Remark 3.2. The same argument that proves (3.2) yields a canonical isomorphism of étale sheaves on $S$

$$
j_{*} \underline{X}_{*}(T)=\underline{\operatorname{Hom}}_{S}\left(j_{*} \underline{X}^{*}(T), j_{*} \mathbb{Z}_{K}\right) .
$$

Let

$$
v_{T}: \mathscr{T} \rightarrow i_{*}\left(\underline{X}^{*}(T)^{I}\right)^{\vee}
$$

be the epimorphism of étale sheaves which appears in the exact sequence of Lemma 3.1. If $T=\mathbb{G}_{m, K}$, then $v_{T}=v_{\mathbb{G}_{m, K}}: j_{*} \mathbb{G}_{m, K} \rightarrow i_{*} \mathbb{Z}_{k}$ is the morphism appearing 
in the exact sequence (2.6). For arbitrary $T$, and via the identifications (3.2) and (3.3), $v_{T}$ is the morphism

$$
\underline{\operatorname{Hom}}_{S}\left(j_{*} \underline{X}^{*}(T), v_{\mathbb{G}_{m, K}}\right): \underline{\operatorname{Hom}}_{S}\left(j_{*} \underline{X}^{*}(T), j_{*} \mathbb{G}_{m, K}\right) \rightarrow \underline{\operatorname{Hom}}_{S}\left(j_{*} \underline{X}^{*}(T), i_{*} \mathbb{Z}_{k}\right) .
$$

Now, since $\left(\underline{X}^{*}(T)^{I}\right)^{\vee}=i^{*} i_{*}\left(\underline{X}^{*}(T)^{I}\right)^{\vee}$ by [29, Proposition II.8.1.1, p. 134], the exact sequence of Lemma 3.1 induces an exact sequence of étale $k$-sheaves

$$
0 \longrightarrow i^{*} \underline{\operatorname{Hom}}_{S}\left(j_{*} \underline{X}^{*}(T), \mathbb{G}_{m, S}\right) \longrightarrow i^{*} \mathscr{T} \stackrel{i^{*} v_{T}}{\longrightarrow}\left(\underline{X}^{*}(T)^{I}\right)^{\vee} \longrightarrow 0 .
$$

Since (2.3) is an equivalence, $i^{*} v_{T}: i^{*} \mathscr{T} \rightarrow\left(\underline{X}^{*}(T)^{I}\right)^{\vee}$ corresponds to a homomorphism of $k$-group schemes $\mathscr{T}_{s} \rightarrow E$, where $E$ is the étale $k$-group scheme that represents $\left(\underline{X}^{*}(T)^{I}\right)^{\vee}$ (see [29, Proposition II.9.2.3, p. 153]). By diagram (2.4), the latter homomorphism factors (uniquely) through a homomorphism of $k$-group schemes $\pi_{0}\left(\mathscr{T}_{s}\right) \rightarrow E$. Thus there exists a commutative diagram of étale $k$-sheaves

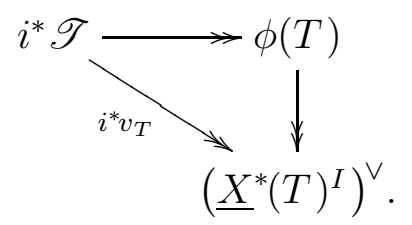

Let

$$
\alpha_{T}: \phi(T) \rightarrow\left(X^{*}(T)^{I}\right)^{\vee}
$$

be the epimorphism of $g$-modules which corresponds to the vertical morphism in (3.5).

Proposition 3.3. The map

$$
\alpha_{T}^{\vee}: X^{*}(T)^{I} \hookrightarrow \phi(T)^{\vee}
$$

induced by (3.6) is an isomorphism of g-modules.

Proof. See [5, Theorem 5.1.6, p. 93] and note that the g-module $E(T)=$ Coker $\alpha_{T}^{\vee}$ appearing there vanishes if $k$ is perfect by [5, Theorem 5.3.8, p. 104].

Corollary 3.4. The map (3.6) induces an isomorphism of g-modules

$$
\phi(T) / \phi(T)_{\text {tors }} \stackrel{\sim}{\rightarrow}\left(X^{*}(T)^{I}\right)^{\vee} .
$$

Proof. This is immediate from the proposition and (2.8).

Lemma 3.5. If $H^{1}\left(I, X^{*}(T)\right)=0$, then there exists a canonical isomorphism of g-modules

$$
\beta_{T}: \phi(T) \stackrel{\sim}{\rightarrow} X_{*}(T)_{I}
$$

Proof. By [31, Proposition 2.7], $\phi(T)$ is torsion free. Thus, by Corollary (3.4), $\alpha_{T}: \phi(T) \rightarrow\left(X^{*}(T)^{I}\right)^{\vee}$ is an isomorphism of g-modules. On the other hand, by (3.1), $q_{T}: X_{*}(T)_{I} \rightarrow\left(X^{*}(T)^{I}\right)^{\vee}$ is an isomorphism of $g$-modules. Thus $\beta_{T}:=$ $q_{T}^{-1} \circ \alpha_{T}: \phi(T) \rightarrow X_{*}(T)_{I}$ is the required isomorphism of $g$-modules. 
We note that, if $T$ has multiplicative reduction (i.e., $I$ acts trivially on $X^{*}(T)$ ), then the isomorphism of the lemma is the isomorphism (2.15). Further, since the isomorphism of the previous lemma is canonical (i.e., functorial in $T$ ), given a morphism of $K$-tori $T_{1} \rightarrow T_{2}$ such that $H^{1}\left(I, X^{*}\left(T_{1}\right)\right)=H^{1}\left(I, X^{*}\left(T_{2}\right)\right)=0$, the induced diagram

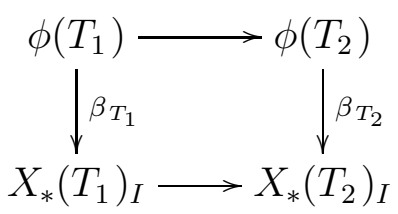

commutes.

Now recall the canonical resolution (2.21)

$$
0 \rightarrow P \rightarrow Q \rightarrow T \rightarrow 0,
$$

where $P$ and $Q$ are given by (2.19) and (2.20), respectively. Since $P$ has multiplicative reduction, we have $H^{1}\left(I, X^{*}(P)\right)=0$. Further, $H^{1}\left(I, X^{*}(Q)\right)=0$ by Lemma 2.7. Thus, by (3.7) and Lemmas 2.5, 2.6 and 3.5, there exists a canonical exact commutative diagram

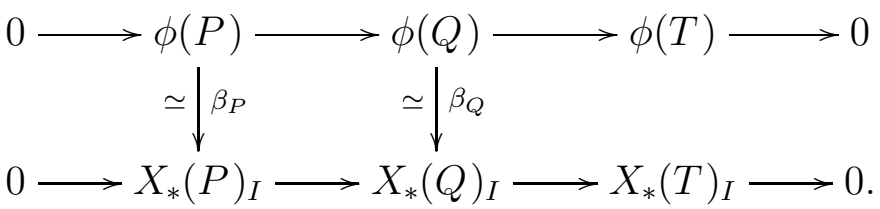

It is now clear that there exists a unique isomorphism of $g$-modules $\beta_{T}: \phi(T) \stackrel{\sim}{\rightarrow}$ $X_{*}(T)_{I}$ such that the following diagram, derived from (3.8),

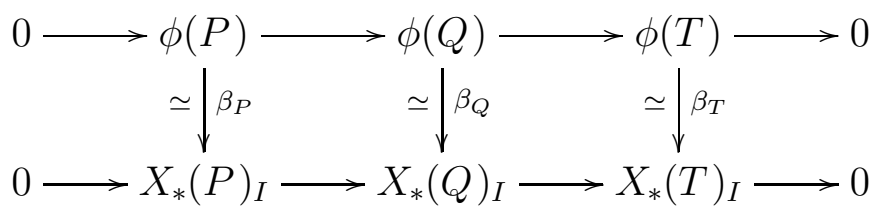

commutes.

The isomorphism thus defined fits into a commutative diagram

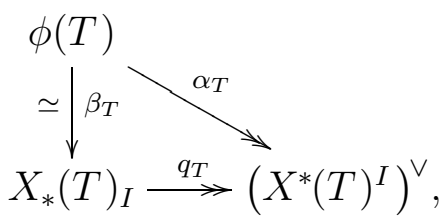

where $q_{T}$ and $\alpha_{T}$ are the epimorphisms given by (3.1) and (3.6), respectively.

The proof of Theorem 1.1 is now complete.

The following consequence of Theorem 1.1 was previously established in [31, Corollary 2.18]. 
Corollary 3.6. There exists a canonical exact sequence of g-modules

$$
0 \rightarrow H^{1}\left(I, X^{*}(T)\right)^{D} \rightarrow \phi(T) \rightarrow\left(X^{*}(T)^{I}\right)^{\vee} \rightarrow 0 .
$$

In particular, $\phi(T)_{\text {tors }}$ is canonically isomorphic to $H^{1}\left(I, X^{*}(T)\right)^{D}$.

Proof. This follows from Theorem 1.1 together with (3.1).

Remark 3.7. By Lemma 2.4 and the corollary, $T$ has unipotent reduction if, and only if, $\phi(T)$ is finite. If this is the case, then there exists a canonical isomorphism of finite $g$-modules $\phi(T)=H^{1}\left(I, X^{*}(T)\right)^{D}$.

The following result clarifies the exactness properties of the functor $\phi(-)$.

Proposition 3.8. Let $0 \rightarrow T_{1} \rightarrow T_{2} \rightarrow T_{3} \rightarrow 0$ be an exact sequence of $K$-tori. Then the given sequence of $K$-tori induces an exact sequence of $g$-modules

$0 \rightarrow H^{2}\left(I, X^{*}\left(T_{1}\right)\right)^{D} \rightarrow H^{2}\left(I, X^{*}\left(T_{2}\right)\right)^{D} \rightarrow H^{2}\left(I, X^{*}\left(T_{3}\right)\right)^{D} \rightarrow \phi\left(T_{1}\right) \rightarrow \phi\left(T_{2}\right) \rightarrow \phi\left(T_{3}\right) \rightarrow 0$.

Proof. The exactness of the sequence $0 \rightarrow H^{2}\left(I, X^{*}\left(T_{1}\right)\right)^{D} \rightarrow H^{2}\left(I, X^{*}\left(T_{2}\right)\right)^{D} \rightarrow$ $H^{2}\left(I, X^{*}\left(T_{3}\right)\right)^{D}$, which is induced by the short exact sequence of $I$-modules $0 \rightarrow$ $X^{*}\left(T_{3}\right) \rightarrow X^{*}\left(T_{2}\right) \rightarrow X^{*}\left(T_{1}\right) \rightarrow 0$, follows from the fact that $H^{3}\left(I, X^{*}\left(T_{3}\right)\right)=0$ since $K^{\mathrm{nr}}$ has cohomological dimension $\leq 1$ (see [26, II, beginning of $\S 4.3$, p. 85]). On the other hand, by the right exactness of the functor (from $\mathcal{G}$-modules to $g$ modules) $M \mapsto M_{I}$ (see [30, Exercise 6.1.1(2), p. 160]), the short exact sequence of $\mathcal{G}$-modules $0 \rightarrow X_{*}\left(T_{1}\right) \rightarrow X_{*}\left(T_{2}\right) \rightarrow X_{*}\left(T_{3}\right) \rightarrow 0$ induces an exact sequence of $g$-modules $X_{*}\left(T_{1}\right)_{I} \rightarrow X_{*}\left(T_{2}\right)_{I} \rightarrow X_{*}\left(T_{3}\right)_{I} \rightarrow 0$. By Theorem 1.1, the latter sequence can be identified with a sequence $\phi\left(T_{1}\right) \rightarrow \phi\left(T_{2}\right) \rightarrow \phi\left(T_{3}\right) \rightarrow 0$. Now the connecting homomorphism $H^{2}\left(I, X^{*}\left(T_{3}\right)\right)^{D} \rightarrow \phi\left(T_{1}\right)=X_{*}\left(T_{1}\right)_{I}$ in the sequence of the proposition factors as $H^{2}\left(I, X^{*}\left(T_{3}\right)\right)^{D} \rightarrow H^{1}\left(I, X^{*}\left(T_{1}\right)\right)^{D} \hookrightarrow X_{*}\left(T_{1}\right)_{I}$ (see (3.1)), and its kernel is therefore equal to the kernel of $H^{2}\left(I, X^{*}\left(T_{3}\right)\right)^{D} \rightarrow H^{1}\left(I, X^{*}\left(T_{1}\right)\right)^{D}$, i.e., to the image of $H^{2}\left(I, X^{*}\left(T_{2}\right)\right)^{D} \rightarrow H^{2}\left(I, X^{*}\left(T_{3}\right)\right)^{D}$. It remains only to check exactness at $\phi\left(T_{1}\right)$. By [5, Theorem 2.3.4, p. 52], the kernel of $\phi\left(T_{1}\right) \rightarrow \phi\left(T_{2}\right)$ is a finite $g$-module, whence it agrees with

$$
\operatorname{Ker}\left[\phi\left(T_{1}\right)_{\text {tors }} \rightarrow \phi\left(T_{2}\right)_{\text {tors }}\right]=\operatorname{Ker}\left[H^{1}\left(I, X^{*}\left(T_{1}\right)\right)^{D} \rightarrow H^{1}\left(I, X^{*}\left(T_{2}\right)\right)^{D}\right]
$$

(see Corollary 3.6). Since the latter group agrees with the image of $H^{2}\left(I, X^{*}\left(T_{3}\right)\right)^{D} \rightarrow$ $H^{1}\left(I, X^{*}\left(T_{1}\right)\right)^{D}$, the proof is complete.

The following corollary of the proposition generalizes Lemma 2.3.

Corollary 3.9. Let $0 \rightarrow T_{1} \rightarrow T_{2} \rightarrow T_{3} \rightarrow 0$ be an exact sequence of $K$-tori. If $\phi\left(T_{1}\right)$ is torsion-free, then the induced sequence of g-modules

$$
0 \rightarrow \phi\left(T_{1}\right) \rightarrow \phi\left(T_{2}\right) \rightarrow \phi\left(T_{3}\right) \rightarrow 0 .
$$

is exact. 
Proof. As seen in the above proof, the connecting homomorphism $H^{2}\left(I, X^{*}\left(T_{3}\right)\right)^{D} \rightarrow$ $\phi\left(T_{1}\right)$ in the exact sequence of the proposition factors through $H^{1}\left(I, X^{*}\left(T_{1}\right)\right)^{D}=$ $\phi\left(T_{1}\right)_{\text {tors }}=0$.

Remark 3.10. Let $0 \rightarrow T_{1} \rightarrow T_{2} \rightarrow T_{3} \rightarrow 0$ be as in the corollary, i.e., $\phi\left(T_{1}\right)$ is torsion-free. Further, for $i=1,2$ and 3 , let $\mathscr{T}_{i}$ denote the Néron model of $T_{i}$ over $S$. By the corollary and [21, Theorem II.2.15, p. 63], $0 \rightarrow i_{*} \phi\left(T_{1}\right) \rightarrow i_{*} \phi\left(T_{2}\right) \rightarrow$ $i_{*} \phi\left(T_{3}\right) \rightarrow 0$ is an exact sequence of étale sheaves on $S$. On the other hand, since $\mathrm{R}^{1} j_{*} T_{1}=0$ for the étale topology on $S$ (see [31, Lemma 2.3]), $0 \rightarrow \mathscr{T}_{1} \rightarrow \mathscr{T}_{2} \rightarrow$ $\mathscr{T}_{3} \rightarrow 0$ is an exact sequence of étale sheaves on $S$. Thus there exists a canonical exact commutative diagram of étale sheaves on $S$

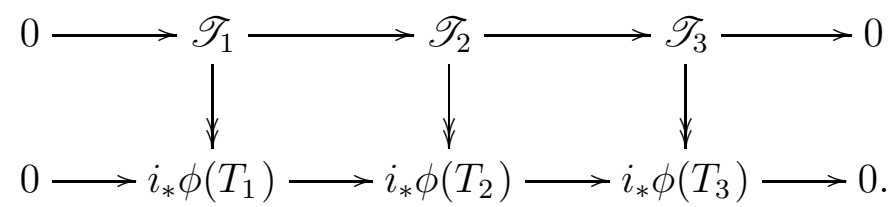

By (2.5), the above diagram yields an exact sequence $0 \rightarrow \mathscr{T}_{1}^{0} \rightarrow \mathscr{T}_{2}^{0} \rightarrow \mathscr{T}_{3}^{0} \rightarrow 0$ of étale sheaves on $S$ and therefore an exact sequence of (representable) étale $k$-sheaves

$$
0 \rightarrow i^{*} \mathscr{T}_{1}^{0} \rightarrow i^{*} \mathscr{T}_{2}^{0} \rightarrow i^{*} \mathscr{T}_{3}^{0} \rightarrow 0 .
$$

Since (2.3) is an equivalence, the latter sequence corresponds to a sequence of smooth, affine, commutative and connected algebraic $k$-group schemes

$$
0 \rightarrow \mathscr{T}_{1, s}^{0} \stackrel{f}{\rightarrow} \mathscr{T}_{2, s}^{0} \stackrel{g}{\rightarrow} \mathscr{T}_{3, s}^{0} \rightarrow 0 .
$$

The exactness of (3.9) implies that $g$ is surjective and $f$ identifies $\mathscr{T}_{1, s}^{0}$ with Ker $g:=$ $\mathscr{T}_{2, s}^{0} \times \mathscr{T}_{3, s}^{0}$ Spec $k$. See, for example, [1, Lemma 2.1]. Now [1, Lemma 2.5] shows that the sequence of representable presheaves on Spec $k$ induced by (3.10) is an exact sequence of fppf and fpqc sheaves on Spec $k$. In other words, (3.10) is exact for the étale, fppf and fpqc topologies on Spec $k$.

\section{The Cohomology of Tori}

All cohomology groups below are taken with respect to the étale topology on the relevant scheme.

Let $T$ be a $K$-torus. The maximal subtorus $T_{(m)}$ of $T$ having multiplicative reduction is the $K$-torus with character module $N X^{*}(T)$ (see [23, Proposition 1.2]). On the other hand, the maximal quotient torus $T^{(u)}$ of $T$ having unipotent reduction is the $K$-torus with character module ${ }_{N} X^{*}(T)$. Indeed, if $Y$ is a $\mathcal{G}$-submodule of $X^{*}(T)$ such that $Y^{I}=Y^{J}=0$, then $N Y=0$, i.e., $Y \subset{ }_{N} X^{*}(T)$. Now the exact sequence of $\mathcal{G}$-modules $0 \rightarrow{ }_{N} X^{*}(T) \rightarrow X^{*}(T) \rightarrow N X^{*}(T) \rightarrow 0$ induces an exact sequence of $K$-tori

$$
0 \rightarrow T_{(m)} \rightarrow T \rightarrow T^{(u)} \rightarrow 0
$$


Let $\mathscr{T}_{(m)}, \mathscr{T}$ and $\mathscr{T}^{(u)}$ denote, respectively, the Néron models of $T_{(m)}, T$ and $T^{(u)}$ over $S$. Since $X^{*}\left(T_{(m)}\right)=N X^{*}(T)$ is torsion-free, Remark 3.10 yields a sequence of smooth, affine, commutative and connected algebraic $k$-groups

$$
0 \rightarrow \mathscr{T}_{(m), s}^{0} \rightarrow \mathscr{T}_{s}^{0} \rightarrow\left(\mathscr{T}^{(u)}\right)_{s}^{0} \rightarrow 0
$$

The latter sequence is exact for the étale, fppf and fpqc topologies on Spec $k$. Set

$$
\tau=\mathscr{T}_{(m), s}^{0,},
$$

which is the unique maximal $k$-torus of $\mathscr{T}_{s}^{0}$. Note that the $g$-module of characters of $\tau$ is $N X^{*}(T)$. In particular, if $T$ has multiplicative reduction, so that $J=1$ and $N X^{*}(T)=X^{*}(T)$, then the character module of $\tau$ is $X^{*}(T)$ regarded as a g-module.

Lemma 4.1. For every $r \geq 1$, there exists a canonical isomorphism of abelian groups $H^{r}\left(k, \mathscr{T}_{s}^{0}\right)=H^{r}(k, \tau)$.

Proof. Since $k$ is perfect, the sequence (4.2) splits (see [10, XVII, Theorem 6.1.1]), i.e., there exists an isomorphism of $k$-group schemes $\mathscr{T}_{s}^{0} \simeq \tau \times U$, where $U=$ $\left(\mathscr{T}^{(u)}\right)_{s}^{0}$. Since $U$ is algebraic, smooth, connected and unipotent, it has a composition series whose successive quotients are $k$-isomorphic to $\mathbb{G}_{a, k}$ (see [10, XVII, Corollary 4.1.3]). Thus, since $H^{r}\left(k, \mathbb{G}_{a}\right)=0$ for every $r \geq 1$ by [25, Chapter X, $\S 1$, Proposition 1, p. 150], we have $H^{r}(k, U)=0$ for every $r \geq 1$. The lemma is now clear.

Now, since $\mathrm{R}^{s} j_{*} T=0$ for the étale topology on $S$ for all $s>0$ by [31, Lemma 2.3], the Leray spectral sequence in étale cohomology $H^{r}\left(S, \mathrm{R}^{s} j_{*} T\right) \Longrightarrow H^{r+s}(K, T)$ yields isomorphisms $H^{r}(S, \mathscr{T})=H^{r}\left(S, j_{*} T\right)=H^{r}(K, T)$ for every $r \geq 0$. On the other hand, $H^{r}\left(S, \mathscr{T}^{0}\right)=H^{r}\left(k, \mathscr{T}_{s}^{0}\right)=H^{r}(k, \tau)$ for every $r \geq 1$ by Lemma 4.1 and [15, Theorem 11.7]. Further, $H^{r}\left(S, i_{*} \phi(T)\right)=H^{r}\left(k, i^{*} i_{*} \phi(T)\right)=H^{r}(k, \phi(T))$ for every $r \geq 0$ by [22, Proposition II.1.1(b), p.149]. Thus, by (2.5), there exists a canonical exact sequence of abelian groups

$$
\cdots \rightarrow H^{r}(k, \tau) \rightarrow H^{r}(K, T) \rightarrow H^{r}(k, \phi(T)) \rightarrow \ldots
$$

where $r \geq 1$ and $\tau$ is the $k$-torus (4.3). By Theorem 1.1, the preceding sequence is canonically isomorphic to a sequence

$$
\cdots \rightarrow H^{r}(k, \tau) \rightarrow H^{r}(K, T) \rightarrow H^{r}\left(k, X_{*}(T)_{I}\right) \rightarrow \ldots
$$

We now derive some consequences of (4.4) and (4.5).

Proposition 4.2. Assume that $T$ has unipotent reduction. Then, for every $r \geq 1$, there exists a canonical isomorphism of abelian groups

$$
H^{r}(K, T)=H^{r}\left(k, H^{1}\left(I, X^{*}(T)\right)^{D}\right) .
$$

Proof. This is clear from (4.4) and Remark 3.7 since $\tau=0$ in this case.

The next proposition generalizes [21, Example III.2.22(c), p. 108] (at least when the ring $A$ appearing in [loc.cit.] is complete). 
Proposition 4.3. Assume that $T$ has multiplicative reduction. Then, for every $r \geq 1$, the sequence of abelian groups induced by (4.5)

$$
0 \rightarrow H^{r}(k, \tau) \rightarrow H^{r}(K, T) \rightarrow H^{r}\left(k, X_{*}(T)\right) \rightarrow 0
$$

is split exact.

Proof. Since $I$ acts trivially on $X_{*}(T)$, (3.4) is a morphism $v_{T}: \mathscr{T} \rightarrow i_{*}\left(\underline{X^{*}}(T)^{I}\right)^{\vee}=$ $i_{*} \underline{X}_{*}(T)$ and the map $H^{r}(K, T) \rightarrow H^{r}\left(k, X_{*}(T)\right)$ appearing in the sequence of the proposition can be identified with the homomorphism $H^{r}\left(v_{T}\right): H^{r}(S, \mathscr{T}) \rightarrow$ $H^{r}\left(S, i_{*} \underline{X}_{*}(T)\right)$ induced by $v_{T}$. Recall also that, via the identifications (3.2) and (3.3), $v_{T}$ can be identified with the morphism

$$
\underline{\operatorname{Hom}}_{S}\left(j_{*} \underline{X}^{*}(T), v_{\mathbb{G}_{m, K}}\right): \underline{\operatorname{Hom}}_{S}\left(j_{*} \underline{X}^{*}(T), j_{*} \mathbb{G}_{m, K}\right) \rightarrow \underline{\operatorname{Hom}}_{S}\left(j_{*} \underline{X}^{*}(T), i_{*} \mathbb{Z}_{k}\right),
$$

where $v_{\mathbb{G}_{m, K}}: j_{*} \mathbb{G}_{m, K} \rightarrow i_{*} \mathbb{Z}_{k}$ is the morphism appearing in the exact sequence (2.6). Now choose a uniformizer $\pi \in A$, let $u: \mathbb{Z}_{K} \rightarrow \mathbb{G}_{m, K}$ be the homomorphism of $K$-group schemes which maps $1 \in \mathbb{Z}$ to $\pi \in \mathbb{G}_{m, K}(K)=K^{*}$ and let $u_{\mathbb{G}_{m, K}}: j_{*} \mathbb{Z}_{K} \rightarrow j_{*} \mathbb{G}_{m, K}$ be the morphism of étale sheaves on $S$ induced by $u$. By Remark 3.2 , there exists a canonical isomorphism (of étale sheaves on $S$ ) $j_{*} \underline{X}_{*}(T)=$ $\underline{\operatorname{Hom}}_{S}\left(j_{*} \underline{X}^{*}(T), j_{*} \mathbb{Z}_{K}\right)$. Thus

$$
\underline{\operatorname{Hom}}_{S}\left(j_{*} \underline{X}^{*}(T), u_{\mathbb{G}_{m, K}}\right): \underline{\operatorname{Hom}}_{S}\left(j_{*} \underline{X}^{*}(T), j_{*} \mathbb{Z}_{K}\right) \rightarrow \underline{\operatorname{Hom}}_{S}\left(j_{*} \underline{X}^{*}(T), j_{*} \mathbb{G}_{m, K}\right)
$$

can be identified with a morphism $u_{T}: j_{*} \underline{X}_{*}(T) \rightarrow \mathscr{T}$. Clearly, the morphism of étale sheaves on $S$

$$
v_{T} \circ u_{T}: j_{*} \underline{X}_{*}(T) \rightarrow i_{*} \underline{X}_{*}(T)
$$

can be identified with $\underline{\operatorname{Hom}}_{S}\left(j_{*} \underline{X}^{*}(T), v_{\mathbb{G}_{m, K}} \circ u_{\mathbb{G}_{m, K}}\right)$, where $v_{\mathbb{G}_{m, K}} \circ u_{\mathbb{G}_{m, K}}: j_{*} \mathbb{Z}_{K} \rightarrow$ $i_{*} \mathbb{Z}_{k}$. On the other hand, by [29, Proposition II.8.2.1, p. 142], for any étale sheaf $\mathscr{F}$ on $S$ there exists a canonical exact exact sequence of étale sheaves on $S$

$$
0 \rightarrow j ! j^{*} \mathscr{F} \rightarrow \mathscr{F} \rightarrow i_{*} i^{*} \mathscr{F} \rightarrow 0
$$

Setting $\mathscr{F}=j_{*} \underline{X}_{*}(T)$ above and noting that $j^{*} j_{*} \underline{X}_{*}(T)=\underline{X}_{*}(T)$ and $i^{*} j_{*} \underline{X}_{*}(T)=$ $\underline{X}_{*}(T)$, we obtain a canonical exact sequence of étale sheaves on $S$

$$
0 \longrightarrow j_{!} \underline{X}_{*}(T) \longrightarrow j_{*} \underline{X}_{*}(T) \stackrel{w_{T}}{\longrightarrow} i_{*} \underline{X}_{*}(T) \longrightarrow 0 \text {. }
$$

It is immediate that $v_{\mathbb{G}_{m, K}} \circ u_{\mathbb{G}_{m, K}}=w_{\mathbb{G}_{m, K}}$, which implies that $v_{T} \circ u_{T}=w_{T}$ for any $T$. On the other hand, since $H^{r}\left(S, j_{!} \underline{X}_{*}(T)\right)=0$ for every $r \geq 1$ by [22, Proposition II.1.1(a), p. 149], the map $H^{r}\left(w_{T}\right): H^{r}\left(S, j_{*} \underline{X}_{*}(T)\right) \rightarrow H^{r}\left(S, i_{*} \underline{X}_{*}(T)\right)$ induced by $w_{T}$ is an isomorphism for every $r \geq 1$ and we conclude that $H^{r}\left(u_{T}\right) \circ H^{r}\left(w_{T}\right)^{-1}$ is a section (i.e., right inverse) of $H^{r}\left(v_{T}\right)$.

The following lemma is well-known but we were unable to find an appropriate reference.

Lemma 4.4. Assume that $k$ has cohomological dimension $\leq 1$. If $\tau$ is a $k$-torus, then $H^{r}(k, \tau)=0$ for every $r \geq 1$. 
Proof. Since $k$ is perfect, $k$ is a field of dimension $\leq 1$ by [26, Chapter II, §3.1, Proposition 6(b), p. 78]. Thus, by [26, Chapter II, §3.1, Proposition 5(iii), p. 78], for any finite Galois extension $l / k, l^{*}$ is a cohomologically trivial $\mathrm{Gal}(l / k)$-module. Let $l$ be the minimal splitting field of $\tau$. Then, since $X^{*}(\tau)$ is a free (and therefore projective) $\mathbb{Z}$-module, we have $\operatorname{Ext}_{\mathbb{Z}}^{1}\left(X^{*}(\tau), l^{*}\right)=0$, whence $\tau(l)=\operatorname{Hom}\left(X^{*}(\tau), l^{*}\right)$ (see (2.2) ) is cohomologically trivial as well by [25, Chapter IX, §5, Theorem 9, p. 145]. Finally, since $H^{r}(k, \tau)$ is the inductive limit of the groups $H^{r}(\mathrm{Gal}(l / k), \tau(l))$ as $l / k$ ranges over the set of all finite Galois subextensions of $\bar{k} / k$, the proof is complete.

Theorem 4.5. Assume that $k$ has cohomological dimension $\leq 1$. Then

(i) The sequence $0 \rightarrow \mathscr{T}^{0}(A) \rightarrow T(K) \rightarrow \phi(T)(k) \rightarrow 0$ is exact.

(ii) For $r=1$ and 2, there exist canonical isomorphisms

$$
H^{r}(K, T) \simeq H^{r}\left(k, X_{*}(T)_{I}\right)
$$

If $r \geq 3$, the groups $H^{r}(K, T)$ vanish.

Proof. The last assertion follows from (4.4) since $H^{r}(k, \tau)=H^{r}(k, \phi(T))=0$ for $r \geq 3$. Assertions (i) and (ii) are immediate from (4.4), (4.5) and Lemma 4.4.

Remark 4.6. If $k$ has cohomological dimension $\leq 1$, then $H^{r}\left(k, H^{1}\left(I, X^{*}(T)\right)^{D}\right)=0$ for $r \geq 2$ since $H^{1}\left(I, X^{*}(T)\right)^{D}$ is a finite $g$-module. Thus, by (3.1) and assertion (ii) of the theorem,

$$
H^{2}(K, T)=H^{2}\left(k, X_{*}(T)_{I}\right)=H^{2}\left(k,\left(X^{*}(T)^{I}\right)^{\vee}\right) .
$$

The preceding remark is a particular case of the following proposition.

Proposition 4.7. Assume that $k$ has finite cohomological dimension $n \geq 1$. Then there exists a canonical isomorphism of divisible abelian groups

$$
H^{n+1}(K, T)=H^{n+1}\left(k,\left(X^{*}(T)^{I}\right)^{\vee}\right) .
$$

If $r \geq n+2$, the groups $H^{r}(K, T)$ vanish.

Proof. The group on the right above is divisible by [27, Corollary 1, p. 55]. Now, since $\tau(\bar{k})$ is divisible, we have $H^{r}(k, \tau)=0$ for every $r \geq n+1$ by [27, Proposition 14, p. 54]. Thus (4.5) yields a canonical isomorphism of abelian groups $H^{r}(K, T)=$ $H^{r}\left(k, X_{*}(T)_{I}\right)$ for every $r \geq n+1$. On the other hand, since $H^{1}\left(I, X^{*}(T)\right)^{D}$ is finite, we have $H^{r}\left(k, H^{1}\left(I, X^{*}(T)\right)^{D}\right)=0$ for all $r \geq n+1$ and (3.1) yields isomorphisms $H^{r}\left(k, X_{*}(T)_{I}\right)=H^{r}\left(k,\left(X^{*}(T)^{I}\right)^{\vee}\right)$ for each $r \geq n+1$. The latter group vanishes if $r \geq n+2$ by [27, Proposition 14, p. 54], whence the proposition follows. 


\section{Abelian Cohomology of Reductive Groups}

Assume that $k$ has cohomological dimension $\leq 1$. Recall that a $K$-torus $F$ is called flasque if the $\mathcal{G}$-module $X_{*}(F)$ is $H^{1}$-trivial (see [8, Lemma 1(iv), p. 179]). By [9, Proposition-Definition 3.1, p. 88, and Proposition 2.2, p. 86], any connected reductive algebraic group $G$ over $K$ admits a flasque resolution, i.e., there exists a central extension

$$
1 \rightarrow F \rightarrow H \rightarrow G \rightarrow 1
$$

where $F$ is a flasque $K$-torus and $H$ is a connected reductive algebraic over $K$ such that the derived group $H^{\text {der }}$ of $H$ is simply connected and $R:=H^{\text {tor }}:=H / H^{\text {der }}$ is a quasi-trivial $K$-torus. The finitely generated (continuous) $\mathcal{G}$-module $\pi_{1}(G):=$ Coker $\left[X_{*}(F) \rightarrow X_{*}(R)\right]$ is independent (up to isomorphism) of the choice of resolution (5.1) and is called the algebraic fundamental group of $G$. See [9, PropositionDefinition 6.1, p. 102]. Recall from [13, Corollary 4.3] that, if $r \geq 1$ is an integer, the $r$-th (flat) abelian cohomology group of $G$ may be defined as the flat hypercohomology group

$$
H_{\mathrm{ab}}^{r}\left(K_{\mathrm{fl}}, G\right):=\mathbb{H}^{r}\left(K_{\mathrm{fpp}}, \pi_{1}(G) \otimes \mathbb{L}^{\mathbf{L}} \mathbb{G}_{m, K}\right) .
$$

These abelian groups are of interest because they can be related to the pointed Galois cohomology sets $H^{r}(K, G)$ for $r=1$ and 2. See Corollary 5.2 below for the case $r=1$.

Now, since $R$ is quasi-trivial, we have $H^{1}(K, R)=0$. Further, $H^{r}(K, F)=0$ for every $r \geq 3$ by the last assertion of Theorem [4.5. Thus, by [13, Proposition 4.2], $H_{\mathrm{ab}}^{r}\left(K_{\mathrm{fl}}, G\right)=0$ for every $r \geq 3$ and (5.1) induces an exact sequence of abelian groups

$$
0 \rightarrow H_{\mathrm{ab}}^{1}\left(K_{\mathrm{fl}}, G\right) \rightarrow H^{2}(K, F) \rightarrow H^{2}(K, R) \rightarrow H_{\mathrm{ab}}^{2}\left(K_{\mathrm{f}}, G\right) \rightarrow 0 .
$$

Now, by [9, Proposition 6.2, p. 102], there exists a canonical exact sequence of $\mathcal{G}$-modules

$$
0 \rightarrow X_{*}(F) \rightarrow X_{*}(R) \rightarrow \pi_{1}(G) \rightarrow 0
$$

which induces a short exact sequence of $g$-modules

$$
0 \rightarrow X_{*}(F)_{I} / M \rightarrow X_{*}(R)_{I} \rightarrow \pi_{1}(G)_{I} \rightarrow 0,
$$

where $M$ is a finite submodule of $X_{*}(F)_{I}$ which is isomorphic to a quotient of $H_{1}\left(J, \pi_{1}(G)\right)$ (see the proof of Lemma 2.6). Since $H^{r}(k, M)=0$ for every $r \geq 2$, we have $H^{2}\left(k, X_{*}(F)_{I} / M\right)=H^{2}\left(k, X_{*}(F)_{I}\right)$ and $H^{r}\left(k, X_{*}(F)_{I} / M\right)=0$ for $r \geq 3$ (see [27, Proposition 14, p. 54]). Further, $H^{1}\left(k, X_{*}(R)_{I}\right)=H^{1}(K, R)=0$ by Theorem 4.5(ii). Thus (5.4) induces an exact sequence of abelian groups

$$
0 \rightarrow H^{1}\left(k, \pi_{1}(G)_{I}\right) \rightarrow H^{2}\left(k, X_{*}(F)_{I}\right) \rightarrow H^{2}\left(k, X_{*}(R)_{I}\right) \rightarrow H^{2}\left(k, \pi_{1}(G)_{I}\right) \rightarrow 0 .
$$


Now consider the exact commutative diagram

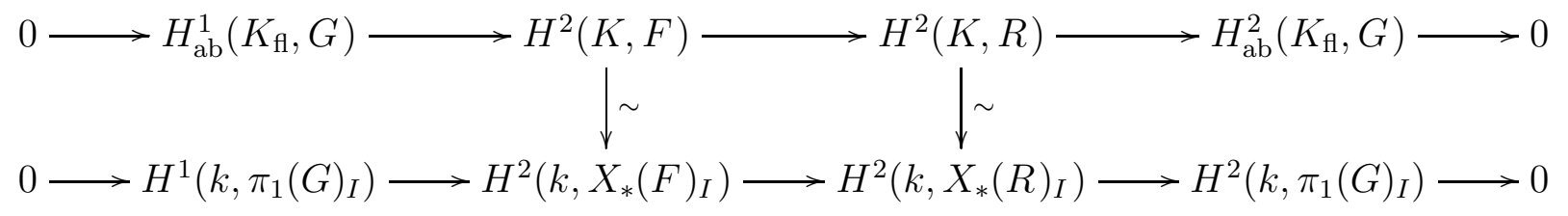

whose top and bottom rows are the sequences (5.2) and (5.5), respectively, and vertical maps are the isomorphisms of Theorem 4.5(ii). Clearly, the maps $H^{2}(K, F) \stackrel{\sim}{\rightarrow}$ $H^{2}\left(k, X_{*}(F)_{I}\right)$ and $H^{2}(K, R) \stackrel{\sim}{\rightarrow} H^{2}\left(k, X_{*}(R)_{I}\right)$ induce isomorphisms

$$
H_{\mathrm{ab}}^{r}\left(K_{\mathrm{fl}}, G\right) \stackrel{\sim}{\rightarrow} H^{r}\left(k, \pi_{1}(G)_{I}\right)
$$

for $r=1$ and 2. Thus the following holds.

Theorem 5.1. Assume that $k$ has cohomological dimension $\leq 1$ and let $G$ be a connected reductive algebraic group over $K$. Then the flasque resolution (5.1) induces isomorphisms of abelian groups

$$
H_{\mathrm{ab}}^{r}\left(K_{\mathrm{ff}}, G\right) \simeq H^{r}\left(k, \pi_{1}(G)_{I}\right)
$$

for $r=1$ and 2 . If $r \geq 3$, the groups $H_{\mathrm{ab}}^{r}\left(K_{\mathrm{f}}, G\right)$ vanish.

Corollary 5.2. Assume that $k$ has cohomological dimension $\leq 1$. Then there exists a bijection of pointed sets

$$
H^{1}(K, G) \simeq H^{1}\left(k, \pi_{1}(G)_{I}\right) .
$$

In particular, $H^{1}(K, G)$ can be endowed with an abelian group structure.

Proof. Let $\widetilde{G}$ be the simply connected central cover of $G^{\text {der }}$ (see [13, p. 1161] for the definition of $\widetilde{G}$ ). By [6, Theorem 4.7(ii), p. 697, and Remark 3.16(3), p. 695], the Galois cohomology set $H^{1}(K, \widetilde{G})$ is trivial. Further, by [12, VII, Theorem 3.1, p. 99], $K$ is a field of Douai type in the sense of [14, Definition 5.2]. Thus, by [14. Theorem 5.8(i)], the first abelianization map ab ${ }^{1}: H^{1}(K, G) \rightarrow H_{\mathrm{ab}}^{1}\left(K_{\mathrm{fl}}, G\right)$ is bijective. The corollary is now immediate from the theorem.

Corollary 5.3. Assume that $k$ is quasi-finite. Then there exists a bijection of pointed sets $H^{1}(K, G) \simeq \pi_{1}(G)_{\mathcal{G} \text {,tors }}$.

Proof. Since a quasi-finite field is perfect and of cohomological dimension $\leq 1$ by [25, XIII, beginning of $\S 2$, p. 190] and [27, III, §2, Corollary 3, p. 69], the corollary is immediate from the previous corollary and the following lemma.

Lemma 5.4. Assume that $k$ is quasi-finite and let $M$ be a continuous g-module. Then there exists a canonical isomorphism of abelian groups $H^{1}(k, M) \simeq M_{g \text {,tors }}$. 
Proof. Let $\sigma$ be a free generator of g. By [25, XIII, §1, Proposition 1, p. 189], there exists a canonical isomorphism of abelian groups $H^{1}(k, M)=M^{\prime} /(\sigma-1) M$, where $M^{\prime}$ is the subgroup of $M$ consisting of those $x \in M$ for which there exists an integer $n \geq 1$ such that $\left(1+\sigma+\cdots \sigma^{n-1}\right) x=0$. Thus it remains only to check that $M^{\prime} /(\sigma-1) M$ is the full torsion subgroup of $M_{g}=M /(\sigma-1) M$. Let $x \in M$ be such that $m x=(\sigma-1) y$ for some positive integer $m$ and some $y \in M$ and choose a positive integer $r$ such that $\sigma^{r} x=x$ and $\sigma^{r} y=y$. Then

$$
\begin{aligned}
\left(1+\sigma+\cdots \sigma^{m r-1}\right) x & =\left(1+\sigma+\cdots \sigma^{r-1}\right)\left(1+\sigma^{r}+\cdots \sigma^{(m-1) r}\right) x \\
& =\left(1+\sigma+\cdots \sigma^{r-1}\right) m x=\left(1+\sigma+\cdots \sigma^{r-1}\right)(\sigma-1) y \\
& =\left(\sigma^{r}-1\right) y=0 .
\end{aligned}
$$

\section{Remarks 5.5.}

(a) When $K$ is a finite extension of $\mathbb{Q}_{p}$, Corollary 5.3 is due to Borovoi. See [3, Corollary 5.5(i)].

(b) Assume that $k$ has finite cohomological dimension $n \geq 1$ and let $\mu$ be the kernel of the canonical morphism $\widetilde{G} \rightarrow G$. Then $\mu$ is a finite and commutative $K$-group scheme. By [26, §II.4.3, Proposition 12, p. 85] and [28, Theorem 4, p. 593], $H^{r}\left(K_{\mathrm{fl}}, \mu\right)=0$ for every $r \geq n+2$. Consequently, the exact sequence in [13, p. 1174, line 8] yields an isomorphism of abelian groups $H_{\mathrm{ab}}^{n+1}\left(K_{\mathrm{fl}}, G\right) \simeq H^{n+1}\left(K, G^{\text {tor }}\right)$. Thus, by the proof of Proposition 4.7, there exists an isomorphism $H_{\mathrm{ab}}^{n+1}\left(K_{\mathrm{fl}}, G\right) \simeq H^{n+1}\left(k, X_{*}\left(G^{\text {tor }}\right)_{I}\right)$. On the other hand, it follows from [9, Proposition 6.4, p. 104] that there exists an isomorphism of g-modules $X^{*}\left(G^{\text {tor }}\right)_{I} \simeq \pi_{1}(G)_{I} / M^{\prime}$, where $M^{\prime}$ is a finite submodule of $\pi_{1}(G)_{I}$. Thus there exists an isomorphism of abelian groups

$$
H_{\mathrm{ab}}^{n+1}\left(K_{\mathrm{fl}}, G\right) \simeq H^{n+1}\left(k, \pi_{1}(G)_{I}\right)
$$

which generalizes the case $r=2$ of Theorem 5.1 .

\section{REFERENCES}

[1] A. Bertapelle and C.D. González-Avilés, The Greenberg functor revisited. arXiv:1311.0051 [math.NT].

[2] R.Bitan, The discriminant of an algebraic torus. Journal of Number Theory 131 (2011), 1657 1671.

[3] M.Borovoi, Abelian Galois cohomology of reductive groups. Memoirs of the American Mathematical Society 132 (1998).

[4] S. Bosch, W. Lütkebohmert and M. Raynaud, Néron models. Springer-Verlag, Berlin 1989.

[5] B. Brahm, Néron-Modelle algebraischer Tori, Schriftenreihe des Mathematisches Instituts der Universität Münster, Vol. 31, Universität Münster, Münster, 2004.

[6] F. Bruhat and J. Tits, Groupes algébriques sur un corps local. Chapitre III. Compléments et applications à la cohomologie galoisienne. Journal of the Faculty of Science. University of Tokyo. Section IA. Mathematics 34 (1987), 671-698. 
[7] H. Cartan and S. Eilenberg, Homological Algebra. Princeton University Press, Princeton, 1956.

[8] J.-L. Colliot-Thélène and J.-J. Sansuc, La $R$-équivalence sur les tores. Annales Scientifiques de l'École Normale Supérieure 10 (1977), 175-229.

[9] J.-L. Colliot-Thélène, Résolutions flasques des groupes linéaires connexes. Journal für die reine und angewandte Mathematik 618 (2008), 77-133.

[10] M. Demazure and A. Grothendieck (Eds.), Schémas en groupes. Séminaire de Géométrie Algébrique du Bois Marie 1962-64 (SGA 3). Augmented and corrected 2008-2011 re-edition of the original by P.Gille and P.Polo. Available at http://www.math.jussieu.fr/ polo/SGA3 Reviewed at http://www.jmilne.org/math/xnotes/SGA3r.pdf.

[11] M. Demazure and P. Gabriel, Groupes algébriques. Tome I: Géométrie algébrique, généralités, groupes commutatifs. Paris: Masson \& Cie, Éditeur, 1970.

[12] J.-C. Douai, 2-Cohomologie Galoisienne des groupes semi-simples, Thèse de doctorat d'etat, Université de Lille, 1976. Éditions universitaires européennes, 2010.

[13] C.D. González-Avilés, Flasque resolutions of reductive group schemes. Central European Journal of Mathematics 11 (2013), 1159-1176.

[14] C.D. González-Avilés, Quasi-abelian crossed modules and nonabelian cohomology. Journal of Algebra 369 (2012), 235-255.

[15] A. Grothendieck, Le groupe de Brauer III. Exemples et compléments. Dix exposés sur la cohomologie de schémas. North-Holland, Amsterdam: Masson, Paris (1968): 88-188.

[16] A. Grothendieck and J. Dieudonné, Éléments de géométrie algébrique I. Le langage des schémas. Grundlehren der Mathematischen Wissenschaften 166 (1971).

[17] A. Grothendieck and J. Dieudonné, Éléments de géométrie algébrique II, Étude globale élémentaire de quelques classes de morphismes. Publications Mathématiques de l'Institut des Háutes Études Scientifiques 8 (1961), 5-222.

[18] A. Grothendieck and J. Dieudonné, Éléments de géométrie algébrique IV, Étude locale des schémas et des morphismes de schémas, Quatrième partie. Publications Mathématiques de l'Institut des Háutes Études Scientifiques 32 (1967), 5-361.

[19] R. Kottwitz, Isocrystals with additional structure. II. Compositio Mathematica 109 (1997), $255-339$.

[20] B. Kunyavskii and B. Moroz, On integral models of algebraic tori and affine toric varieties. Trudy SPMO 13 (2007), 97-119. English translation in Proceedings of the Saint Petersburg Mathematical Society XIII, (2008), 75-92. American Mathematical Society Translations, Series 2, 222, Providence, Rhode Island, U.S.A.

[21] J.S. Milne, Étale Cohomology. Princeton Mathematical Series 33, Princeton University Press, 1980.

[22] J.S. Milne, Arithmetic Duality Theorems, Second Edition. Electronic version, http://www.jmilne.org/math/books/, 2006.

[23] E. Nart and X. Xarles, Additive reduction of algebraic tori, Archiv der Mathematik 57 (1991), 460-466.

[24] G. Pappas and M. Rapoport, Twisted loop groups and their affine flag varieties. With an Appendix by T. Haines and M. Rapoport. Advances in Mathematics 219 (2008), 118-198.

[25] J.-P., Serre, Local Fields. Graduate Texts in Mathematics 67 (second corrected printing, 1995). New York-Berlin: Springer-Verlag, 1979.

[26] J.-P. Serre, Galois Cohomology. Springer Monographs in Mathematics. Berlin: SpringerVerlag, 1997.

[27] S. Shatz, Profinite groups, Arithmetic, and Geometry. Annals of Mathematics Studies 67, Princeton University Press, Princeton 1972. 
[28] S. Shatz, The cohomological dimension of certain Grothendieck topologies. Annals of Mathematics 83 (1966), 572-595.

[29] G. Tamme, Introduction to Étale Cohomology. Universitext. Berlin: Springer-Verlag, 1994.

[30] C. A. Weibel, An introduction to homological algebra. Cambridge Studies in Advanced Mathematics 38, Cambridge University Press, Cambridge 1994.

[31] X. Xarles, The scheme of connected components of the Néron model of an algebraic torus, Journal für die reine und angewandte Mathematik 437 (1993), 167-179.

Dipartimento di Matematica, Università di Padova, via Trieste 63, I-35121 Padova

E-mail address: alessandra.bertapelle@unipd.it

Departamento de Matemáticas, Universidad de La Serena. Cisternas 1200, La Serena 1700000, Chile

E-mail address: cgonzalez@userena.cl 